\title{
Terrestrial atmospheric effects induced by counterstreaming dense interstellar cloud material
}

\author{
A. Yeghikyan ${ }^{1}$ and H. Fahr ${ }^{2}$ \\ 1 Byurakan Astrophysical Observatory, 378433, Byurakan, Armenia \\ e-mail: aray@astro.uni-bonn.de \\ 2 Institute of Astrophysics and Extraterrestrial Research (IAER), University of Bonn, Auf dem Huegel 71, \\ 53121 Bonn, Germany \\ e-mail:hfahr@astro.uni-bonn.de
}

Received 15 April 2004 / Accepted 26 May 2004

\begin{abstract}
The Solar System during its life has travelled more than 10 times through dense interstellar clouds with particle concentrations of $10^{2}-10^{3} \mathrm{~cm}^{-3}$ and more, compressing the heliosphere to heliopause dimensions smaller than 1 AU and thus bringing the Earth in immediate contact with the interstellar matter. For cloud concentrations greater than of $10^{2} \mathrm{~cm}^{-3}$, the flowing interstellar material even at the Earth's orbit remains completely shielded from solar wind protons and would only be subject to solar photoionization processes. We have developed a 2D-two-fluid gas-dynamical numerical code to describe the hydrodynamical behavior of the incoming interstellar gas near the Earth, taking into account both the photoionization and the gravity of the Sun. As we show, the resulting strongly increased neutral hydrogen fluxes ranging from $10^{9}$ to $10^{11} \mathrm{~cm}^{-2} \mathrm{~s}^{-1} \mathrm{cause}$ substantial changes in the terrestrial atmosphere. During the phase of the immersion into the cloud the resulting flux of neutral hydrogen incident on the terrestrial atmosphere in the steady state would be balanced by the upward escape flux of $\mathrm{H}$-atoms and the downward flux of water molecules, which is the product of the atmospheric hydrogen-oxygen chemistry via even-odd reaction schemes. In that case hydrogen acts as a chemical agent to remove oxygen atoms and to cause ozone concentration reductions above $50 \mathrm{~km}$ by a factor of 1.5 at the stratopause to about a factor of 1000 and more at the mesopause. Thus, depending on the specific encounter parameters the high mixing ratio of hydrogen in the Earth's atmosphere may substantially decrease the ozone concentration in the mesosphere and may trigger an ice age of relatively long duration.
\end{abstract}

Key words. ISM: clouds - ISM: atoms - Earth - solar system: general

\section{Introduction}

From time to time, the Solar System on its galactic itinerary encounters various galactic objects, e.g. spiral arms (Leitch \& Vasisht 1998; Shaviv 2003), star clusters and associations (Innanen 1996), galactic diffuse clouds (H I) and giant molecular clouds (GMC) (Talbot \& Newman 1977), etc. Although all encounter probabilities are finite, only a few of them are high enough to make it worthwhile to consider them. All of these mentioned events correspond to different mean travel times of the solar system between consecutive encounters with the corresponding objects, e.g. depending on their distributions in the galactic plane, their sizes and their peculiar velocities. For example, neutral $\mathrm{H}$ I clouds, having a mean number density in the range from 10 to $100 \mathrm{~cm}^{-3}$ and a radius of about a few $\mathrm{pc}$, are objects fairly frequently encountered by the Sun, perhaps over 100 times since its birth $\sim 4.6 \mathrm{Gyr}$ ago. The more dense GMCs, having densities of $10^{3} \mathrm{~cm}^{-3}$ or more, probably must have been encountered by the Sun about 5-10 times (see e.g., Talbot \& Newman 1977). When such events happen (especially in the case of GMCs) the solar wind expansion region must be reduced to small scales, and the flow of solar coronal matter hence must be deflected into the heliotail within distances of less than $1 \mathrm{AU}$. Thus the Earth under these conditions should inevitably be immersed in the direct flow of the cloud material (see Yeghikyan \& Fahr 2003), at least during its upwind orbital passage. Concerning this aspect it is interesting to note that Wimmer-Schweingruber \& Bochsler (2000) have recently given clear hints that gas constituents implanted in cristalline surface layers of lunar soil grains can be taken as a record of encounters with dense interstellar clouds.

The consequences of intensive neutral hydrogen flows and high $\mathrm{H}$-densities at $1 \mathrm{AU}$ for the chemistry in the terrestrial atmosphere must be envisioned as considerable (Fahr 1968; McKay \& Thomas 1978) and have been repeatedly discussed in the literature (see Scherer 2000; Scherer et al. 2002; Frisch et al. 2002, for recent reviews). For example, such an encounter is suspected of possibly triggering global glaciations, depositions of prebiotic material on the primordial Earth, and biomass extinctions. As concerns the climatic changes, McCrea (1975) has emphasized that just such a triggering mechanism is needed to explain ice epochs which are separated by a few hundred million years. Besides, Yabushita \& Allen (1989) 
have pointed out that a passage through the core of a GMC with a density $n \sim 10^{5} \mathrm{~cm}^{-3}$ could give rise to an inflow of hydrogen atoms into the terrestrial atmosphere of up to $10^{12} \mathrm{~cm}^{-2} \mathrm{~s}^{-1}$, which as a consequence may have substantially depleted the oxygen of the Earth's atmosphere by strongly activated $\mathrm{H}_{2} \mathrm{O}$ formation and so for instance caused a longterm bio-mass extinction at the Cretaceous/Tertiary boundary $\sim 65 \mathrm{Myr}$ ago. Also, only such a cataclysmic (not catastrophic) event is compatible with the well known high concentration of iridium deposited in clay layers of these paleogeologic boundaries. Moreover, Yabushita \& Allen (1997) have stressed that the reduced abundance of oxygen contained in ancient amber may also be taken as a record of such an event of atmospheric oxygen depletion 65 Myr ago.

For more quantitative investigations of these events we have developed a 2D-gas dynamic numerical code to describe the hydrodynamic behavior of incoming interstellar gas-dust matter near the Earth, taking into account both the photoionization and the gravity of the Sun (Yeghikyan \& Fahr 2003). A two-fluid version of our model reveals neutral and ionized flow parameters (Yeghikyan \& Fahr 2004). Previously published results (Zank \& Frisch 1999; Frisch et al. 2002) were limited to a consideration of processes outside the so-called accretion radius $R_{\mathrm{g}}=2 G M / V_{\mathrm{i}}^{2}$ ( $V_{\mathrm{i}}$ is the relative velocity between the Sun and the cloud, e.g. Talbot \& Newman 1977) and also did not take photoionization into account. However, the influence both of gravity and photoionization on the neutral flow at the Earth seems to be important for a quantitative estimation of the inflow parameters (Yeghikyan \& Fahr 2003).

Our aim in this paper is to show that the mechanism of Yabushita and Allen concerning the theory of terrestrial atmospheric oxygen depletion (Yabushita \& Allen 1983, 1989, 1997) has severe limitations and might not work even in the (extremely rare) case of an encounter with the core of a GMC. On the other hand we show that one consequence of this mechanism, first mentioned by McKay \& Thomas (1978) and concerning the simultaneous mesospheric ozone reduction, may occur in the much more likely case of an encounter with any part of a gas-dust GMC with a density of at least $1000 \mathrm{~cm}^{-3}$. This is important because cores of GMCs and clumps within GMCs occupy only $\sim 0.1 \%$ and $\sim 1 \%$ of the volume of the GMC, respectively (Allen 2000).

\section{The flow around the heliosphere}

In this paper we again use our hydrodynamic model of the flow of a dense ISM gas phase around the heliosphere, already described in Yeghikyan \& Fahr (2003, 2004), where all the details of the theory and the calculations can be found.

The physical scenario of the interaction of the heliospheric plasma bubble with counterstreaming IC material is as follows: the dense and cold gas-dust material of a GMC sweeps over the heliosphere, is partly photoionized by the Sun and pushes the heliopause inwards to inside the Earth's orbit. At GMC H-atom densities of the order of $10^{3} \mathrm{~cm}^{-3}$ and more, the free passage of a $1 \mathrm{keV}$ proton (i.e. solar wind proton) is restricted by charge exchange reactions to mean free paths of less than $0.03 \mathrm{AU}$ (Yeghikyan \& Fahr 2003). Pressure equilibrium occurs at an upwind distance of less than 1 AU. At such small solar distances of less than 1 AU the cloud's matter, though not subject to charge exchange with solar wind protons, is, however, effectively photoionized. Hence in the first line an interaction of the solar wind protons with the photo-ionized component of the ISM, rather than with the neutral ISM component, occurs. We are interested in the behavior of the flow at the Earth's orbit $r_{\mathrm{E}}$, which in the upwind direction is evidently outside of the compressed heliosphere. Thus the GMC material when approaching heliocentric distances of 0.1-1.0 AU, but yet outside the heliopause, is only subject to solar photoionization processes.

It has been shown (Yeghikyan \& Fahr 2003, 2004), that clouds with concentrations of from a few $10^{2}$ up to $10^{5}$ particles per $\mathrm{cm}^{3}$ and relative velocities $V_{\mathrm{i}}$ of about $20-26 \mathrm{~km} \mathrm{~s}^{-1}$ are able to cause neutral $\mathrm{H}$-atom fluxes near the Earth of $10^{9}-10^{11}$ atoms $/ \mathrm{cm}^{2} \mathrm{~s}$.

A key parameter, $\omega=R_{\mathrm{g}} / \lambda^{*}$, which determines the net density enhancement of neutral matter, is the ratio of the accretion radius $R_{\mathrm{g}}$ to the typical photoionization scale $\lambda^{*}=\beta_{\mathrm{E}} r_{\mathrm{E}}^{2} / V_{\mathrm{i}}$ (Yeghikyan \& Fahr 2003), where $\beta_{\mathrm{E}}=8 \times 10^{-8} \mathrm{~s}^{-1}$ is the photoionization rate for $\mathrm{H}$-atoms at $r_{\mathrm{E}}$. The quantity " $\omega$ " does not depend on the heliocentric distance, but it depends on the relative velocity between the Sun and the cloud and may vary between 5 and 25 for possible values of $V_{\mathrm{i}}$ ranging from 5 to $30 \mathrm{~km} \mathrm{~s}^{-1}$ (Talbot \& Newman 1977). Finally, provided that the most probable values of $V_{\mathrm{i}}$ are $20-26 \mathrm{~km} \mathrm{~s}^{-1}$ (see, e.g., Yeghikyan \& Fahr 2003, and references therein), the GMC material may give rise to a neutral hydrogen flux enhancement by up to 6 orders of magnitude as compared to the present-day values.

In Fig. 1 one example of our two-fluid hydrodynamic model is shown with an initial concentration of the GMC of $1000 \mathrm{~cm}^{-3}$, a relative velocity of $26 \mathrm{~km} \mathrm{~s}^{-1}$ and an initial temperature of $100 \mathrm{~K}$.

The sizes of the hydrogen dissociation and ionization cavities in the upwind direction are about 2.4 AU and 0.61 AU, respectively (Yeghikyan \& Fahr 2004). This means that the flow at $1 \mathrm{AU}$ must be predominantly atomic (non-molecular) with a neutral hydrogen flux of $F_{\mathrm{H}}=3 \times 10^{9} \mathrm{~cm}^{-2} \mathrm{~s}^{-1}$, where an average relative velocity between the circumsolar $\mathrm{H}$-flow and the orbital motion of the Earth on its orbit has been taken of about $40 \mathrm{~km} \mathrm{~s}^{-1}$ (see Fahr et al. 1988; and Fig. 1).

\section{Atmospheric effects}

This highly reactive monoatomic hydrogen could penetrate the upper terrestrial atmosphere up to some height level where the collisional "optical" thickness becomes 1 , and there could be involved in chemical reactions, regulating the abundances of the important atmospheric species, such as oxygen and ozone (McKay \& Thomas 1978). The latter authors have argued that in the middle atmosphere (between 50 and $150 \mathrm{~km}$ above sea-level) the reactions which interchange odd-hydrogen compounds $\left(\mathrm{H}, \mathrm{OH}, \mathrm{HO}_{2}\right)$ are much faster than those which couple odd-hydrogen to even-hydrogen compounds $\left(\mathrm{H}_{2}, \mathrm{H}_{2} \mathrm{O}\right)$. This makes it possible to balance the incident flux of interstellar (atomic/molecular) hydrogen by the upward escape flux of 

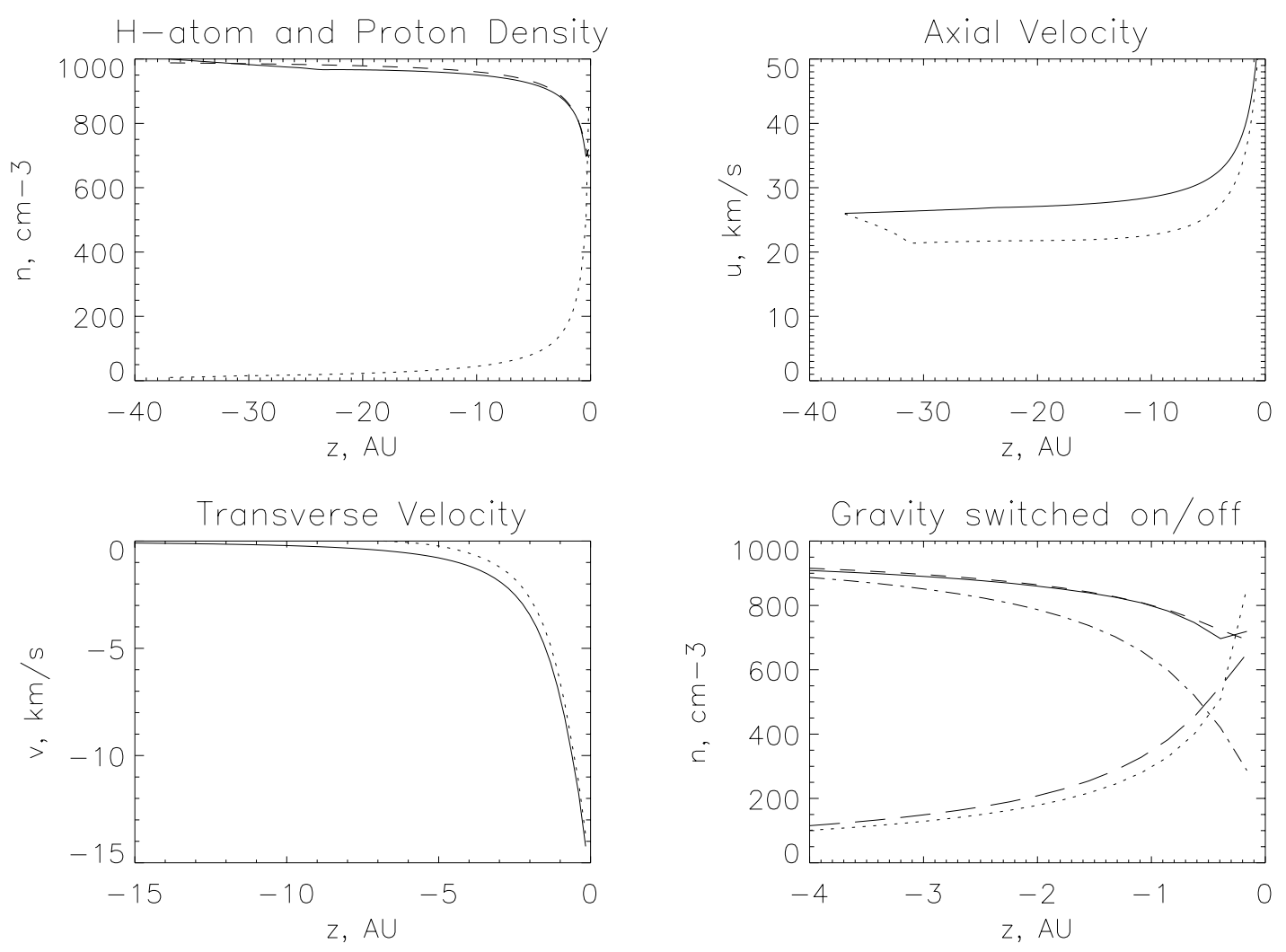

Fig. 1. Number densities, axial and radial components of the velocity of the gas flow versus axial distance $z$, at radial coordinate $r=0$ (the transverse velocities at $r=1 \mathrm{AU}$ ) are shown by solid and dashed curves for the hydrogen atoms and protons, respectively. Here $n_{\mathrm{i}}=$ $1000 \mathrm{~cm}^{-3}, V_{\mathrm{i}}=26 \mathrm{~km} \mathrm{~s}^{-1}$ and $T_{\mathrm{i}}=100 \mathrm{~K}$, respectively. The role of gravity is illustrated in the bottom, right, by solid and dotted (for atoms and protons, $G \neq 0$ ) and by dash-dotted and long-dashed curves (for atoms and protons, $G=0$ ), respectively. For reasons of comparison, the density distributions of hydrogen atoms for the cold kinetic model (e.g., Yeghikyan \& Fahr 2004) are also shown in the top, left and bottom, right, by the dashed curves.

$\mathrm{H}$ atoms and the downward flux of $\mathrm{H}_{2} \mathrm{O}$ in the following form (see McKay \& Thomas 1978):

$$
\begin{aligned}
\Phi & =0.5 \cdot \Phi_{\mathrm{esc}}(\mathrm{H})+\Phi_{\text {down }}\left(\mathrm{H}_{2} \mathrm{O}\right) \\
& =5.9 \times 10^{11} f^{1 / 2}+1.9 \times 10^{13} f .
\end{aligned}
$$

Here $\Phi$ is the flux of incoming interstellar $\mathrm{H}_{2}$, and $f$, as explained in detail by McKay \& Thomas (1978), is the ratio of $\mathrm{H}_{2} \mathrm{O}$ number density to the total atmospheric number density at $100 \mathrm{~km}$ above sea level. Atomic hydrogen atoms move upwards and are lost by exospheric escape while water molecules, which tend to reconstitute the local homospheric density equilibrium distribution, diffuse downward towards the tropospheric levels, form clouds and finally are lost due to water precipitation to the ground. Later Yabushita \& Allen $(1983,1989)$ have shown on the basis of this scheme that all atmospheric oxygen must be removed by interstellar hydrogen in a ratio $2: 1$ by number, if the dense core of a GMC with a density of $10^{5} \mathrm{~cm}^{-3}$ has been encountered by the Earth.

It should be noted that for a complete removal of the present column density $N_{\mathrm{O}}=4.4 \times 10^{43}$ of atmospheric oxygen atoms (in the form of $\mathrm{O}_{2}$ ) it is necessary to have critical fluxes of interstellar hydrogen at $1 \mathrm{AU}$ given by the following simple relation

$F_{\mathrm{c}}=\frac{2 \cdot N_{\mathrm{O}}}{4 \pi R_{\oplus}^{2} \cdot \Delta T}=\frac{1.5 \times 10^{13}}{D(\mathrm{pc})} \frac{\text { atoms }}{\mathrm{cm}^{2} \mathrm{~s}}$, maintained over a certain time period $\Delta T=D / V_{\mathrm{i}}$ which is characteristic for the Solar system passage through a GMC with a dimension $D$. The critical flux level $F_{\mathrm{c}}$ then amounts to $2.9 \times 10^{12}$ and $1.5 \times 10^{12} \mathrm{~cm}^{-2} \mathrm{~s}^{-1}$ for passage times of 0.2 and $0.4 \mathrm{Myr}$ of $5 \mathrm{pc}-$ and $10 \mathrm{pc}-$ sized GMCs, respectively (the relative velocity $V_{\mathrm{i}}$ between the Sun and the GMC is assumed to be $26 \mathrm{~km} \mathrm{~s}^{-1}$ ).

Our scenario described by the two-fluid model predicts a neutral hydrogen flux of $3 \times 10^{9} \mathrm{~cm}^{-2} \mathrm{~s}^{-1}$ for a GMC density of $10^{3} \mathrm{~cm}^{-3}$ (Fig. 1). We assume that under these conditions the incoming neutral hydrogen flow plays the same role as considered in the mentioned even-odd reaction scheme of McKay \& Thomas (1978) for molecular hydrogen, i.e. we assumed that Eq. (1) is valid. In this case, according to Yabushita $\&$ Allen (1989), for a given incoming atomic hydrogen flux $\Phi$, Eq. (1) results in a value of $f$ which in turn yields values for $\Phi_{\text {down }}\left(\mathrm{H}_{2} \mathrm{O}\right)$.

In Fig. 2 we show the incident neutral hydrogen flux, $\Phi$, and the downward flux captured as water, $\Phi_{\text {down }}\left(\mathrm{H}_{2} \mathrm{O}\right)$, versus density of GMC. The results from our hydrodynamical calculations are compared with those of Yabushita \& Allen (1989). A difference is caused by the more refined hydrodynamical and ionizational model which we have used here. The critical fluxes $F_{\mathrm{c}}$ of 5 and $10 \mathrm{pc}$ sized GMC (corresponding to the cases $\mathrm{B}$ and $\mathrm{A}$, with concentrations of $9.1 \times 10^{5}$ and 


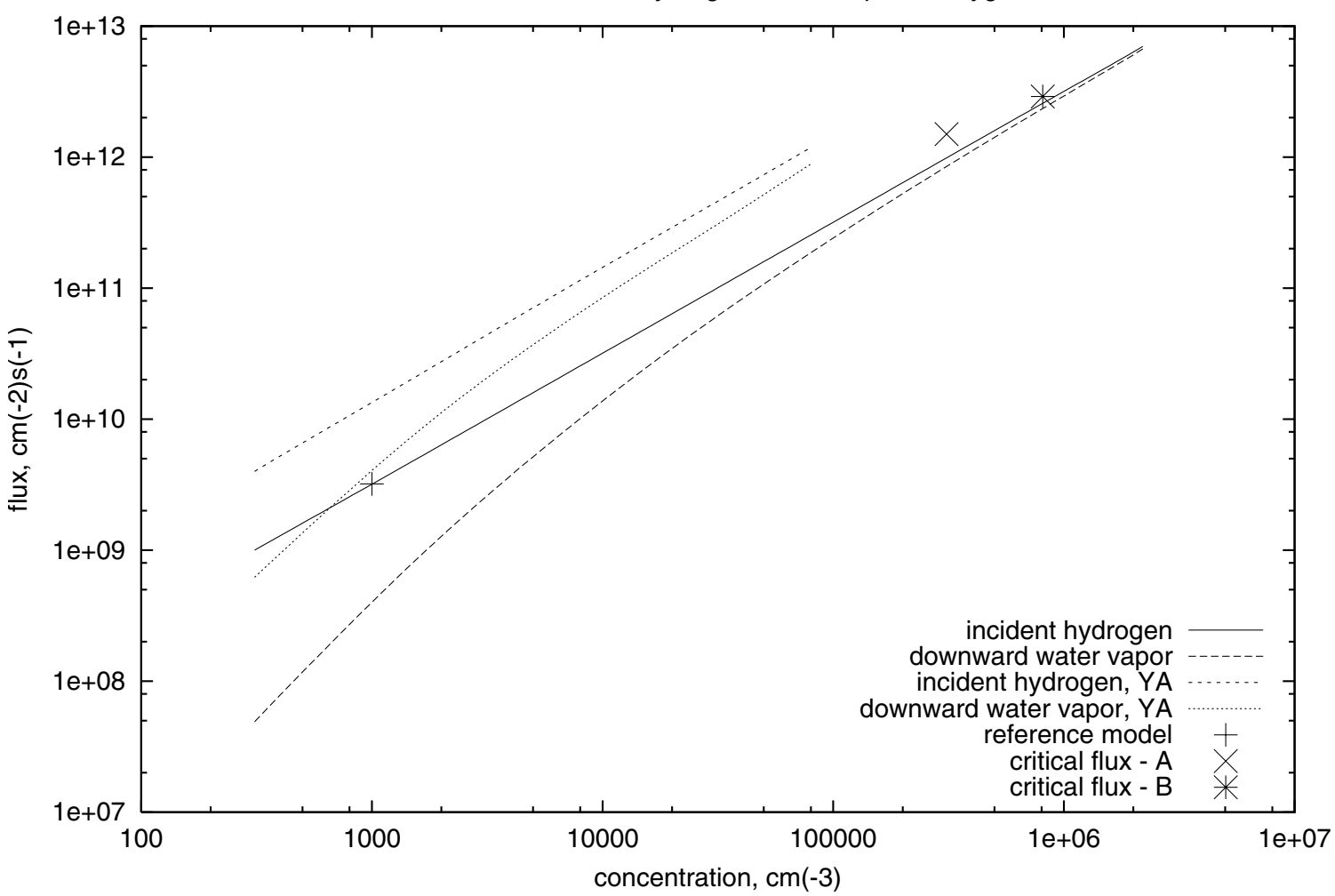

Fig. 2. Flux of incident hydrogen at $1 \mathrm{AU}$, $\Phi$, and downward flux captured as water, $\Phi_{\text {down }}\left(\mathrm{H}_{2} \mathrm{O}\right)$ versus the density of a GMC, are shown by curves marked YA (Yabushita \& Allen's model) and by curves without marks (our hydrodynamical model), respectively. The critical values of incident hydrogen, corresponding to the $5 \mathrm{pc}$ (B) and $10 \mathrm{pc}$ (A) sized GMCs, respectively, are estimated from Eq. (2) and also are shown. The cross is located at the flux level of $3 \times 10^{9} \mathrm{~cm}^{-2} \mathrm{~s}^{-1}$ corresponding to the case of the flow mentioned in Fig. 1 (i.e. $n_{\mathrm{i}}=1000 \mathrm{~cm}^{-3}$ ).

$4.7 \times 10^{5} \mathrm{~cm}^{-3}$, respectively) also are shown. The hydrogen flux level, $F$, due to the case of Fig. 1 is shown by a cross. It is clearly seen that only gaseous material of GMCs with an average density of $5 \times 10^{5} \mathrm{~cm}^{-3}$ or more is sufficient (i.e. $F>F_{\mathrm{c}}$ ) to completely remove all atmospheric oxygen by the mechanism proposed by McKay \& Thomas (1978) and Yabushita \& Allen (1989).

At the same time such densities correspond to cores and clumps of GMCs with dimensions that are one or two orders of magnitude smaller $(D \sim 0.1 \mathrm{pc})$ than those of the whole GMC, i.e. in that case Eq. (2) would require a critical flux that is one or two orders greater than those presented in Fig. 2, due to the shorter times of passage. Thus we conclude that this mechanism should not work for any real GMC with parameters $n \sim 1000 \mathrm{~cm}^{-3}$ and $D \sim 5-10 \mathrm{pc}$.

The remainder of the paper will consist of an attempt to estimate one other consequence of increased hydrogen content in the terrestrial upper atmosphere, particularly its effect on the global ozone concentration.

In the mesosphere, between $50-100 \mathrm{~km}$, the increased influence of hydrogen becomes significant for ozone destruction, as first mentioned by McKay \& Thomas (1978). Note that in general the chemistry of a hydrogen-oxygen-nitrogen atmosphere is governed by very complicated equations. However, when for simplicity the less probable reactions are eliminated from the reaction system, it is possible to develop a general idea of the role of the principal reactions. In particular, since ozone has a short lifetime in the mesosphere and consequently is very close to photochemical equilibrium, its local concentration can conveniently be written as a simple function of water vapor concentration $n\left(\mathrm{H}_{2} \mathrm{O}\right)$ (Nicolet 1971; Chamberlain 1978; Brasseur \& Solomon 1984).

$n\left(\mathrm{O}_{3}\right)=\frac{Y}{\left[n\left(\mathrm{H}_{2} \mathrm{O}\right)\right]^{\alpha}}$.

Here $Y$ denotes a known analytical function depending on the reaction rates involved and on the total and molecular oxygen concentrations at a given altitude. According to Brasseur \& Solomon (1984) $\alpha$ is equal to $1,1 / 2$ and $1 / 3$ at altitudes 80,70 and $50 \mathrm{~km}$, respectively. Ozone is assumed to be formed by three-body recombination between atomic and molecular oxygen and is destroyed by the bi-molecular process, where hydrogen acts as a catalyst, as well as by photodissociation. Again, destruction of odd oxygen (ozone) by $\mathrm{HO}_{x}$-particles $(\mathrm{H}, \mathrm{OH}$, $\mathrm{HO}_{2}$ ) cannot be important below $45 \mathrm{~km}$ (e.g., Crutzen 1971).

Adopting the values of the reaction rates and their distributions with height from the existing atmospheric models (e.g., Hunten \& Donahue 1976, and references therein), the ozone concentration can now be calculated as a function of altitude if the height distribution of water vapor is known. The vertical profile of water vapor is governed, in general, by the competition between its regeneration, its transport and its removal processes. The total downward flux through the mesosphere must be constant because loss and production processes of $\mathrm{H}_{2} \mathrm{O}$ are 


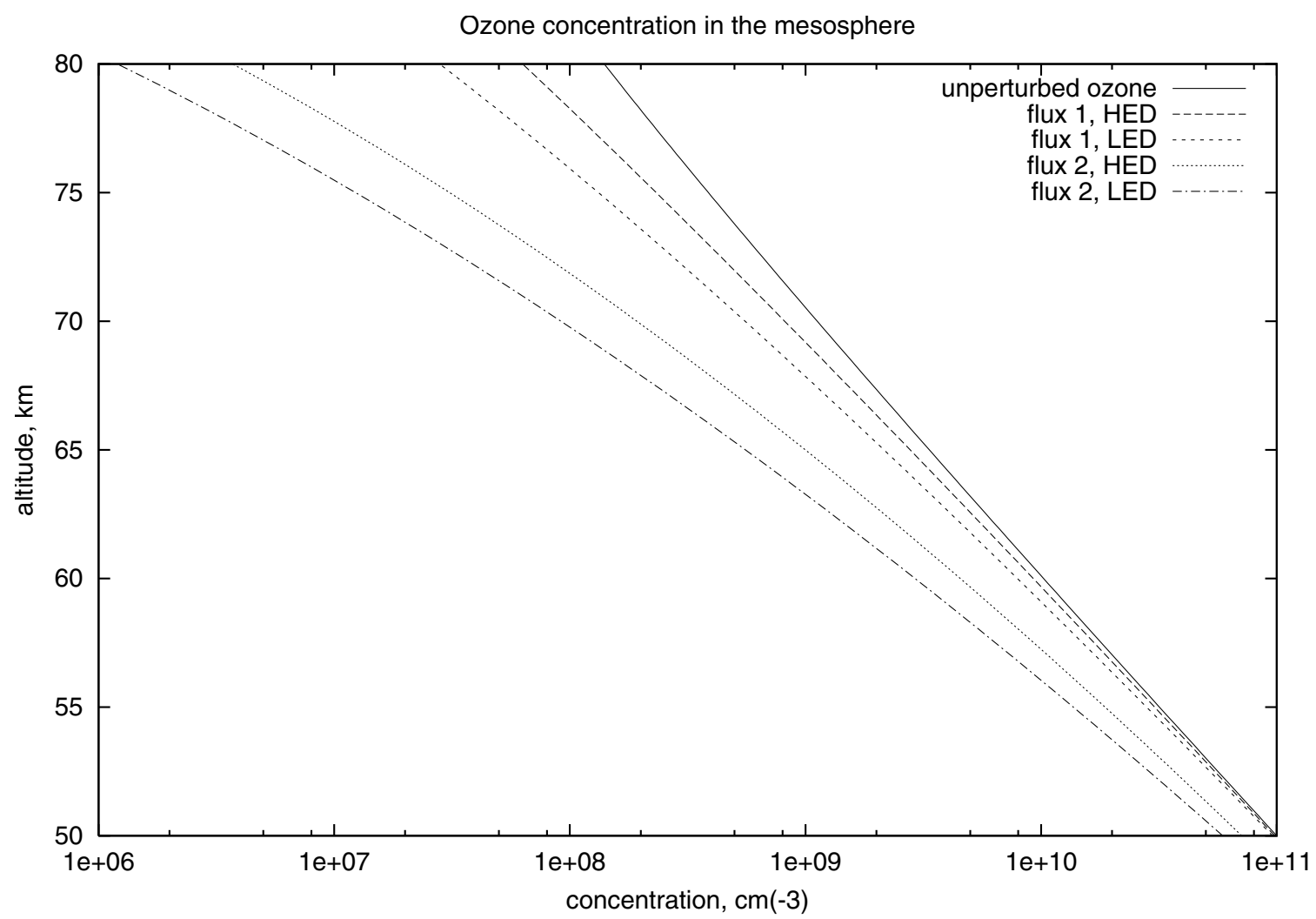

Fig. 3. Distribution of ozone for the unperturbed atmosphere and for two values of incident interstellar hydrogen flux " 1 " $\left(5 \times 10^{9} \mathrm{~cm}^{-2} \mathrm{~s}^{-1}\right)$ and " 2 " $\left(5 \times 10^{10} \mathrm{~cm}^{-2} \mathrm{~s}^{-1}\right)$. The curves labeled HED correspond to the eddy diffusion profile USSA 76 (Brasseur \& Offerman 1986), while the curves labeled LED refer to the profile from Lindzen (1971).

sufficiently rapid (Nicolet 1971). Since the horizontal gradients of the concentrations obviously are relatively small (Brasseur $\&$ Solomon 1984), and since in the mesosphere molecular diffusion is negligible (Hunten \& Donahue 1976), the water vapor height distribution is primarily determined by vertical transport in the eddy-diffusion-controlled regime. Requiring that the downward flux of $\mathrm{H}_{2} \mathrm{O}$ should be constant at a given incoming flux of extraterrestrial hydrogen, one can then relate the value of $\Phi_{\text {down }}\left(\mathrm{H}_{2} \mathrm{O}\right)$ to the concentration of water vapor $n\left(\mathrm{H}_{2} \mathrm{O}\right)$ at the reference altitude of $100 \mathrm{~km}$ (McKay \& Thomas 1978)

$\Phi_{\text {down }}\left(\mathrm{H}_{2} \mathrm{O}\right)=\frac{n\left(\mathrm{H}_{2} \mathrm{O}\right)_{100} \cdot K_{100}}{2 H_{\mathrm{av}}}$,

which in turn gives $n\left(\mathrm{H}_{2} \mathrm{O}\right)$ at the given altitude in the mesosphere

$n\left(\mathrm{H}_{2} \mathrm{O}\right)=\frac{n\left(\mathrm{H}_{2} \mathrm{O}\right)_{100} \cdot K_{100}}{K}$.

$n\left(\mathrm{H}_{2} \mathrm{O}\right)_{100}$ is the perturbed water vapor concentration at $100 \mathrm{~km}$ and $H_{\mathrm{av}}=6 \mathrm{~km}$ is the average mesospheric scale height in the atmosphere (Nicolet 1971).

It must be stressed that the distribution of $\mathrm{H}_{2} \mathrm{O}$ in the mesosphere will strongly depend on the choice made for the vertical profiles of the eddy diffusion coefficients in the calculations of the diffuse downward current. Here the eddy diffusion coefficient at $100 \mathrm{~km}, K_{100}$, is taken to be $2 \times 10^{6} \mathrm{~cm}^{2} \mathrm{~s}^{-1}$, varying with altitude according to the profile proposed in the US Standard Atmosphere (Brasseur \& Offerman 1986). For comparison we will also use the profile proposed by Lindzen (1971), which varies as the square root of density.

Thus the ratio $\delta$ of the ozone concentration $n\left(\mathrm{O}_{3}\right)$ in the mesosphere to its unperturbed value at the same altitude $n^{*}\left(\mathrm{O}_{3}\right)$ is determined by

$\delta=\frac{n\left(\mathrm{O}_{3}\right)}{n\left(\mathrm{O}_{3}\right)^{*}}=\frac{n\left(\mathrm{H}_{2} \mathrm{O}\right)^{\alpha}}{n\left(\mathrm{H}_{2} \mathrm{O}\right)^{* \alpha}}$,

where $\alpha$ is equal to $1,1 / 2$ and $1 / 3$ at altitudes 80,70 and $50 \mathrm{~km}$, respectively, and $n\left(\mathrm{H}_{2} \mathrm{O}\right)^{*}$ is the known unperturbed value of $n\left(\mathrm{H}_{2} \mathrm{O}\right)$.

\section{Results and discussion}

The equilibrium concentrations for perturbed ozone in the mesosphere, between 50 and $100 \mathrm{~km}$, evaluated by means of the adopted unperturbed values from the atmospheric model of Liu \& Donahue (see, e.g., Hunten \& Donahue 1976) are given in Fig. 3 for two values of incoming neutral ISM hydrogen densities, $\sim 2 \times 10^{3} \mathrm{~cm}^{-3}$ and $\sim 2 \times 10^{4} \mathrm{~cm}^{-3}$, respectively.

It can be seen that in all cases a significant reduction of the ozone concentration occurs, owing to the influence of the increased hydrogen fluxes, the total $\mathrm{O}_{3}$ content, as compared with unperturbed values, having decreased from 50-80\% near $80 \mathrm{~km}$ to $2-4 \%$ at the stratopause $(\sim 50 \mathrm{~km})$ and from 2 orders of magnitude $(\sim 80 \mathrm{~km})$ to $30-80 \%(\sim 50 \mathrm{~km})$, for incident fluxes from $5 \times 10^{9} \mathrm{~cm}^{-2} \mathrm{~s}^{-1}$ to $5 \times 10^{10} \mathrm{~cm}^{-2} \mathrm{~s}^{-1}$, respectively. Such fluxes correspond to GMC densities of from $1-2 \times 10^{3}$ to $1-2 \times 10^{4} \mathrm{~cm}^{-3}$, respectively. 
Thus, in the Earth's atmosphere above $45 \mathrm{~km}, \mathrm{H}$-atoms are very important in limiting the natural content of ozone. As a consequence, a lowering of the average height of the mesopause, usually located at an altitude of $85-90 \mathrm{~km}$, as well as a lowering of the average mesopause temperature would be significant. In the perturbed situation there is very little ozone in the neighbouring mesosphere so that hardly any solar UV radiation is absorbed there. In fact, it would penetrate more deeply (by approximately one scale height or $\sim 6 \mathrm{~km}$, see McKay \& Thomas 1978). For this reason, and also because the mesospheric gases at this height radiate considerable heat into space, it is the coldest place in the terrestrial atmosphere and the temperature here decreases and reaches its lowest values. An important consequence is that the creation of saturation conditions over much of the mesosphere should result in a permanent layer of ice clouds of nearly world-wide extent (McKay $\&$ Thomas 1978). In the unperturbed atmosphere such (noctilucent) clouds are formed at high latitudes in summer, in the mesopause region. The analysis (see discussion in McKay \& Thomas 1978, and references therein) reveals that such a cloud layer under perturbed conditions would produce an equilibrium global surface temperature decrease of $1 \mathrm{~K}$. It is now generally recognized that the Earth's climate is very sensitive to small reductions in the surface temperature. In fact, a reduction of $1 \mathrm{~K}$ sustained over a period of several thousand years, is probably enough to trigger an ice age of substantially longer duration. The reason for this sensitivity is the strong positive feedback between temperature and surface albedo, since an ice-covered surface sends much more solar radiation back into space than an ice-free surface (McCrea 1975).

In conclusion, it appears that an enhanced flux of interstellar hydrogen at the terrestrial orbit can produce important climatic effects on the Earth through the complete ozone destruction in the mesosphere. This mechanism may explain, in particular, the ice ages which are separated by roughly a few hundred million years (Yabushita \& Allen 1989).

Other consequences of an enhanced interstellar hydrogen flux for the terrestrial atmosphere, the climate and massextinctions are discussed in McKay \& Thomas (1978) and Yabushita \& Allen (1983, 1985, 1989, 1997).

Acknowledgements. A.Y. was supported financially by the Alexander von Humboldt Foundation and by the Institute of Astrophysics and
Extraterrestrial Research, University of Bonn. The authors are also grateful for financial support granted to them in the frame of the DFGsponsored science project HELIOTRIGGER (Fa-97/28-1), and thank K. Scherer for critical comments.

\section{References}

Allen, C. W. 2000, Astrophysical Quantities, ed. A. N. Cox (Springer) Brasseur, G., \& Offermann, D. 1986, J. Geophys. Res., 91, 10818

Brasseur, G., \& Solomon, S. 1984, Aeronomy of the middle atmosphere (D. Reidel Publ. Comp.)

Chamberlain, J. W. 1978, Theory of planetary atmospheres (Acad. Press.)

Crutzen, P. J. 1971, J. Geophys. Res., 76, 7311

Fahr, H. J. 1968, Astrophys. Space Sci., 2, 474

Fahr, H. J., Grzedzielski, S., \& Ratkiewicz, R. 1988, Ann. Geophys., 6,337

Frisch, P. C., Muller, H. R., Zank, G. P., \& Lopate, C. 2002 [arXiv: astro-ph/0208556]

Hunten, D. M., \& Donahue, T. M. 1976, Ann. Rev. Earth. Planet. Sci., 4, 265

Innanen, K. A. 1996, Earth, Moon and Planets, 72, 1

Leitch, E. M., \& Vasisht, G. 1998, New Astron., 3, 51

Lindzen, R. S. 1971, in Mesospheric models and related experiments, ed. G. Fiocco (D. Reidel Publ.), 122

McCrea, W. H. 1975, Nature, 255, 607

McKay, C. P., \& Thomas, G. E. 1978, Geophys. Res. Lett., 5, 215

Nicolet, M. 1971, in Mesospheric models and related experiments, ed. G. Fiocco (D. Reidel Publ.), 1

Scherer, K. 2000, in The Outer Heliosphere: Beyond the Planets, ed. K. Scherer, H. Fichtner, \& E. Marsch (Copernicus, Katlenburg-Lindau), 327

Scherer, K., Fichtner, H., \& Stawicki, O. 2002, J. Atmosph. Solar-Terr. Phys., 64, 795

Shaviv, N. J. 2003, New Astron., 8, 39

Talbot, R. J., Jr., \& Newman, M. J. 1977, ApJS, 34, 295

Wimmer-Schweingruber, R. F., \& Bochsler, P. 2000, in Acceleration and Transport of Energetic Particles Observed in the Heliosphere, ed. R. A. Mewaldt et al., ACE 2000 Symp., 270

Yabushita, S., \& Allen, A. J. 1983, Observatory, 103, 249

Yabushita, S., \& Allen, A. J. 1985, Observatory, 105, 198

Yabushita, S., \& Allen, A. J. 1989, MNRAS, 238, 1465

Yabushita, S., \& Allen, A. J. 1997, Astron. Geophys., 38(2), 15

Yeghikyan, A. G., \& Fahr, H. J. 2003, Ann. Geophys., 21, 1263

Yeghikyan, A. G., \& Fahr, H. J. 2004, A\&A, 415, 763

Zank, G. P., \& Frisch, P. C. 1999, ApJ, 518, 965 